\title{
Sudden death as presenting symptom caused by cardiac primary multicentric left ventricle rhabdomyoma, in an 11-month-old baby. An immunohistochemical study
}

\author{
Margherita Neri', Sabina Di Donato², Rocco Maglietta ${ }^{3}$, Cristoforo Pomara', Irene Riezzo ${ }^{1}$, Emanuela Turillazzi ${ }^{1}$ \\ and Vittorio Fineschi ${ }^{1 *}$
}

\begin{abstract}
This case report describes a sudden cardiac death in an apparent healthy 11-month-old infant caused by a multifocal cardiac rhabdomyoma. Parents reported that a few days before the child had fallen to the ground getting a little superficial injury to the scalp. The authors hypothesize that it may have been a transient loss of consciousness episode caused by the cardiac tumour. After the gross examination, histological investigation supported by immunohistochemical analysis using antibody anti- Myoglobin, Actin, Vimentin, Desmin, CD34, S-100, Ki-67 was carried out for the diagnosis. Death was attributed to a multifocal cardiac rhabdomyoma, a benign tumour of striated muscle, which has been completely asymptomatic. In particular, one mass filled the entire posterior wall of the left ventricle. The insidious development of benign cardiac tumours also in infants and children is outlined, focusing on the responsible mechanisms of sudden death in such cases and providing a reference for additional study on these subjects.
\end{abstract}

Virtual slides: The virtual slide(s) for this article can be found here:

http://www.diagnosticpathology.diagnomx.eu/vs/7163626988365078

Keywords: Cardiac rhabdomyoma, Sudden death, Cardiac tumours, Paediatric tumours

\section{Background}

Cardiac tumours can be divided into primary and secondary; the first ones occur infrequently both in adult and paediatric age groups. In the general population, their incidence ranges between $0.001 \%$ and $0.030 \%$ in unselected autopsy series; approximately $75 \%$ of primary cardiac tumours are benign, and $25 \%$ are malignant [1]. In adults the most common ones are myxomas.

In the paediatric population, cardiac tumours are reported with a frequency of $0.02 \%$ to $0.04 \%[2,3]$. Usually they are benign, with minimal growth potential, but a minor portion can become quite large, compressing the cardiac chambers or the vital structures such as

\footnotetext{
*Correspondence: vfinesc@tin.it

'Department of Forensic Pathology, University of Foggia, Ospedale Colonnello D'Avanzo, Via degli Aviatori 1, Foggia 71100, Italy

Full list of author information is available at the end of the article
}

conduction tissue or coronary blood vessels, as well as obstructing the cardiac valves and the outflow tracts.

Most paediatric cardiac tumours are considered benign; however, the development of hemodynamic impairment or malignant arrhythmias may complicate the management of these tumours in some patients. In particular, malignant arrhythmias increase the risk of sudden cardiac death [4]. In the absence of these complications, observation is the standard care in most cases because many tumours spontaneously regress with time.

We report the case of a sudden cardiac death of a 11month-old female infant with a primary multicentric left ventricle tumour. Histological and immunohistochemical studies allowed to make the diagnosis of rhabdomyoma and to define the mechanism inducing sudden death. This report outlines the importance of studies performed during the third trimester of pregnancy for early

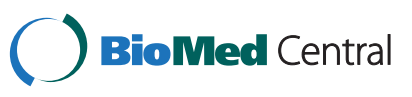


diagnosis of cardiac tumours which, although histologically benign, may be life-threatening for the arrhythmogenic potential they display.

\section{Case presentation}

A mother was bathing her 11-month-old baby, but suddenly the infant showed a worsening dyspnoea. Parents accompanied the baby to the emergency room immediately, but despite the resuscitative manoeuvres, the doctor could only pronounce the death. The familiar history was negative for sudden death. Also the obstetric, remote and recent pathological anamneses were negative, except for a referred unexplained fall dating back to two days before.

A complete post-mortem examination was performed within 48 hours after death. The body was that of a regularly developed 11-month old infant. External examination was insignificant, except for the presence of a little and superficial iatrogenic wound on the sternal region.

The internal examination revealed a peduncolated $4,5 \times 3 \times 2 \mathrm{~cm}$ mass at the cardiac apex, a second superficial subepicardial $2 \times 1,5 \times 1 \mathrm{~cm}$ neoformation on the posterior wall of the left ventricle and a third transmural $1 \times 1,2 \times 1,4 \mathrm{~cm}$ nodule on the posterior wall of the left ventricle (Figure 1). Polyvisceral congestion, cerebral and pulmonary oedema, with a massive increase in lung weight were also evident.

The histological examination of cardiac specimens, stained with haematoxylin-eosin, Masson and PTAH showed a demarcation and separation of the three masses from the surrounding regular parenchyma. The striated muscle cells appeared diffusely vacuolated, enlarged, with round to oval slightly irregular nuclei and variable cytoplasmatic clearing. There were occasional spider cells. Small residues of muscular tissue entrapped in the masses were also visible.

In addition an immunohistochemical investigation of cardiac tissue samples was performed utilizing antibodies anti-Myoglobin, Actin, Vimentin, Desmin, CD 34, Ki67 and S100 (DAKO, Copenhagen, Denmark). For this study, we used $4 \mu \mathrm{m}$-thick paraffin sections mounted on slides covered with 3, aminopropyltriethoxysilane (Fluka, Buchs, Switzerland). Pre-treatment was necessary to facilitate antigen retrieval and to increase membrane permeability to antibodies anti-CD 34 boiling in $0.25 \mathrm{M}$ EDTA buffer, to antibodies anti-Ki67 boiling in $0.1 \mathrm{M}$ Citric Acid buffer, to antibody anti-S100 for $15 \mathrm{~min}$ in Proteolytic Enzyme (Dako, Copenhagen, Denmark), at $20^{\circ} \mathrm{C}$. For antibody anti- Myoglobin, Actin, Vimentin, Desmin it wasn't necessary a pre-treatment for antigen retrieval. The primary antibody was applied in 1:300 ratio for $\mathrm{S100}$, in 1:50 ratio for $\mathrm{CD} 34$, Desmin and Vimentin, in 1:2000 ratio for Actin, in 1:6000 ratio for Myoglobin, in 1:100 ratio for Ki67 and incubated for 120 $\min$ at $20^{\circ} \mathrm{C}$. The detection system utilized was the LSAB+ kit (Dako, Copenhagen, Denmark), a refined avidin-biotin technique in which a biotinylated secondary antibody reacts with several peroxidase-conjugated streptavidin molecules. The sections were counterstained with Mayer's haematoxylin, dehydrated, cover slipped and observed in a Leica DM4000B optical microscope (Leica, Cambridge, UK).

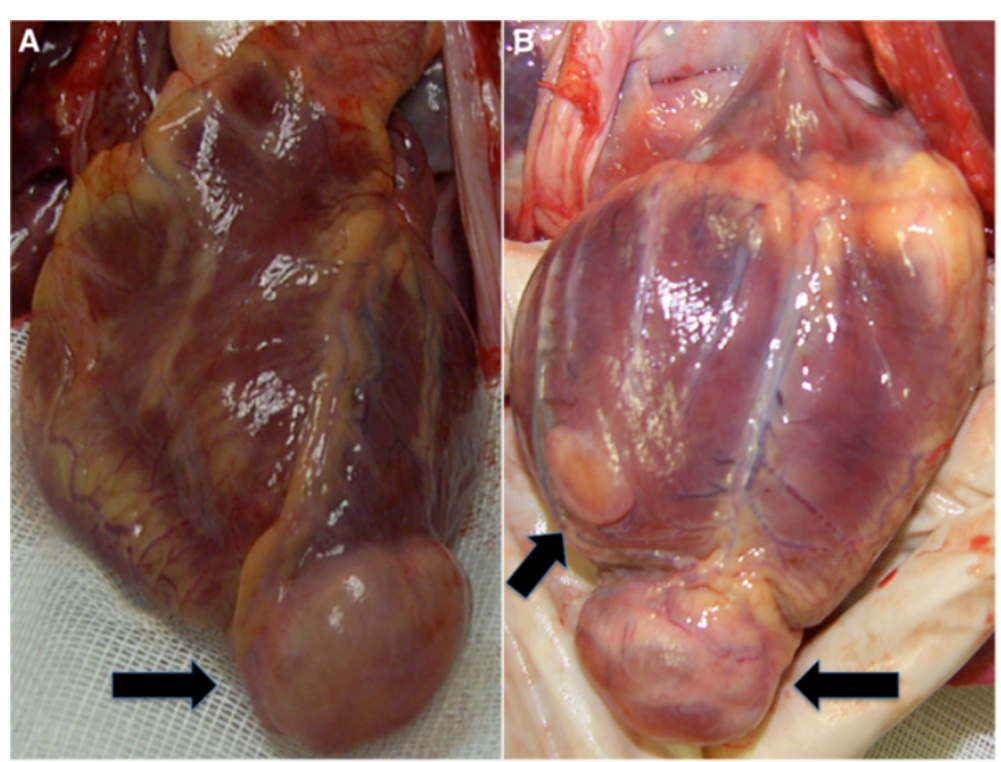

Figure 1 Microscopic findings. The peduncolated mass at the cardiac apex measured $4,5 \times 3 \times 2 \mathrm{~cm}(\mathbf{A})$. A second superficial subepicardial neoformation on the posterior wall of the left ventricle, measured $2 \times 1,5 \times 1 \mathrm{~cm}(\mathbf{B})$. 
The immunohistochemical studies showed an intense positive reaction for antibodies anti-Myoglobin, Actin, Vimentin, Desmin, CD34; while reaction for antibodies Anti-Ki67 and Anti-S100 was negative (Figures 2-3). These results of the immunohistochemical analysis were consistent with the diagnosis of rhabdomyoma.

Toxicological screening resulted negative. There were no signs of tuberous sclerosis.

It was concluded that the sudden cardiac death of the infant was attributable to the multifocal primary cardiac tumour, the rhabdomyoma, consisting in three cardiac lesions. In particular one tumoral mass occupied almost the whole posterior wall of the left ventricle, rising from the apex to the valvular level, so compromising the regular contraction of the left ventricle. The neoplasms probably have caused two days before a near syncopal episode that the parents erroneously referred as a fall.

\section{Conclusions}

Cardiac tumours are infrequent clinical entities both in adult and paediatric age groups; they can be divided into primary and secondary; approximately $75 \%$ of primary cardiac tumours are benign, and 25\% are malignant [1]. The occurrence of metastatic cardiac tumours has been reported 100 -fold more commonly than primary lesions. Approximately half of benign cardiac tumours in adults are myxomas, and the rest are lipomas, papillary fibroelastomas, and rhabdomyomas. Among malignant primary cardiac tumours, the most reported are those histopathologically considered as undifferentiated, followed by angiosarcomas and leiomyosarcomas.
Cardiac tumours in children are rare. It is difficult to estimate the real prevalence because the available data are restricted to autopsy and case records from leading paediatric centres. It is believed that they constitute less than $0.1 \%$ of all neoplasms and occur with an incidence of 0.0017 to 0.28 in autopsy records $[5,6]$.

Among 27.640 patients examined echocardiographically in 5-year periods, the incidence of cardiac tumours increased: $0.06 \%$ (1980-1984), 0.22\% (1985-1989) and $0.32 \%$ (1990-1995) of all the subjects [7].

Benign lesions usually predominate, making up more than $90 \%$ of all paediatric tumours: rhabdomyomas are the most frequent cardiac tumours in children (45-50\%), followed by fibromas (25-30\%), mixomas, lipomas, teratomas, hemangiomas, etc. [8-10]. The most common malignant primary tumours are angiosarcoma, fibrosarcoma, lymphosarcoma, and giant cell sarcoma [2]. Secondary cardiac neoplasms in the paediatric population include non-Hodgkin lymphoma, leukemic infiltration, and neuroblastoma.

Rhabdomyoma is the most common cardiac tumour in paediatric patients. It usually presents during the first few days after birth, but it can be diagnosed in the third trimester of pregnancy.

Foetal cardiac rhabdomyoma accounts for less than $10 \%$ of foetal demise cases [11]. Cardiac rhabdomyomas are benign from the cardiovascular standpoint in most affected foetuses. They are associated strongly with tuberous sclerosis, a hereditary disorder characterized by hamartomas in various organs, epilepsy, mental deficiency, and sebaceous adenomas [12]. Rhabdomyoma (especially multiple) affects about $50 \%$ of children

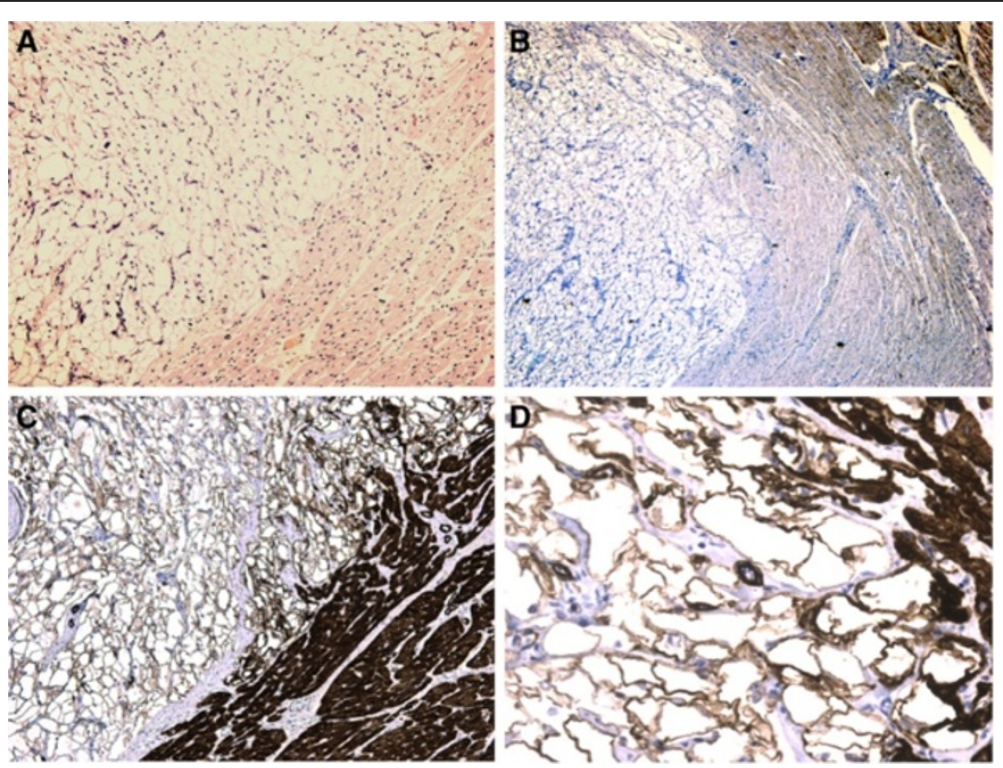

Figure 2 Microscopic findings. (A) Demarcation and separation of the tumoral mass from the surrounding regular parenchyma. Small residues of muscular tissue entrapped in the masses were also visible. Intense positive reaction for antibodies anti-Desmin (B), anti-Actin (C-D). 


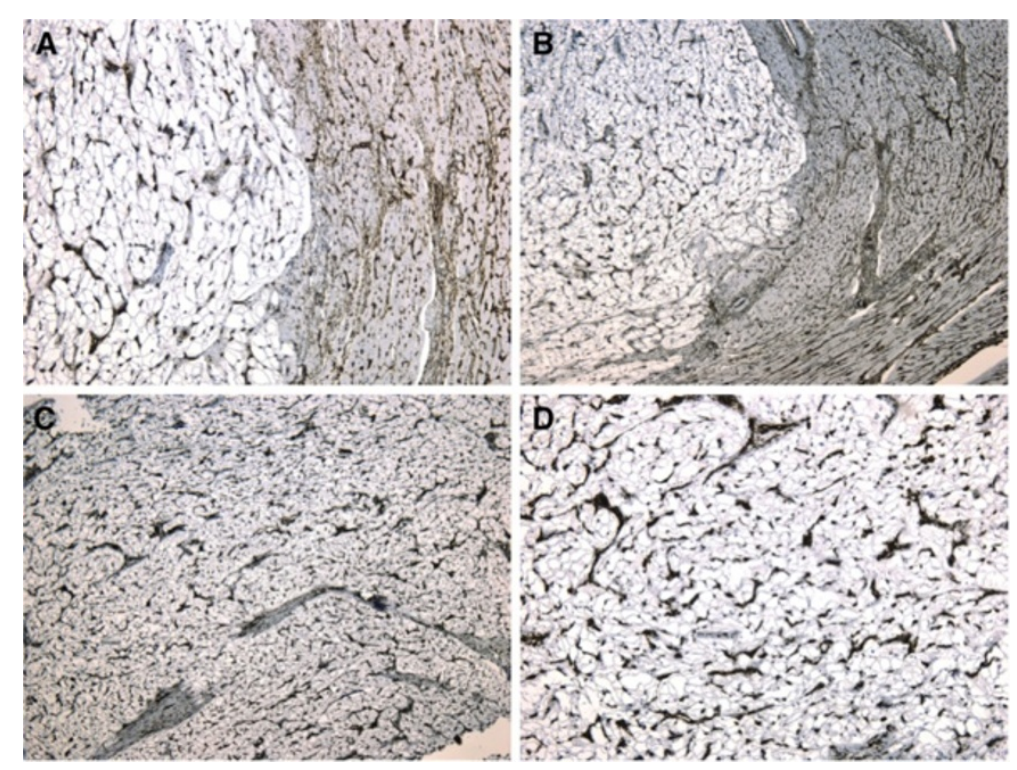

Figure 3 Microscopic findings. (A-B) Intense positive reaction of the tumoral fibers for antibodies anti-CD34 and anti-Vimentin (C-D).

suffering from tuberous sclerosis, but more than $50 \%$ of patients with rhabdomyoma have or will develop tuberous sclerosis. The exceptional patient is one with a solitary, single rhabdomyoma who does not have or develop tuberous sclerosis.

Over $90 \%$ of rhabdomyomas are multiple and occur with approximately equal frequency in both ventricles. The atrium is involved in fewer than $30 \%$ of patients.

Macroscopically, these tumours are firm, grey, and nodular and tend to project into the ventricular cavity. Microscopy shows myocytes of twice normal size filled with glycogen and containing hyperchromatic nuclei and eosinophilic-staining cytoplasmic granules. Scattered bundles of myofibrils can be seen within cells by electron microscopy.

These tumours grow during the second half of pregnancy; they have the tendency to diminish or even completely disappear spontaneously after birth. Many will disappear entirely; alternatively, the tumour size remains constant as the heart grows, which has much the same effect $[13,14]$.

In the majority of cases cardiac rhabdomyomas are clinically completely silent and have a benign course. They may be incidentally discovered during an echocardiogram, but they also may cause cardiac dysfunctions requiring medical and/or surgical intervention. During foetal life and the early neonatal period, life-threatening conditions, mostly due to arrhythmias, cardiac failure or obstruction, do occur on rare occasions [15]. How threatening rhabdomyomas are, in terms of clinical presentation and presence of hemodynamic complications, depends on their size, location and number of lesions. Although the behaviour of cardiac rhabdomyoma is benign, its positioning within critical areas in the heart can lead to lethal arrhythmias and chamber obstruction. The most common presentation is heart failure caused by tumour obstruction of cardiac chambers or valvular orifice flow. Clinical findings may mimic valvular or subvalvular stenosis. Arrhythmias, particularly ventricular tachycardia and sudden death, may be a presenting symptom [16,17]. Atrial tumours may produce atrial arrhythmias. The mechanism of their causing sudden death is likely rhythm disturbances, particularly the Wolff-Parkinson-White ventricular pre-excitation syndrome [17]. The conduction abnormalities and the arrhythmia are thought to be due to the tumoral mass, which may lead to degeneration of the cardiac conduction system and compose a substrate for re-entrant arrhythmia, e.g. the development of atrioventricular block and ventricular arrhythmia. Large tumoral mass in the myocardial region, accompanied with arrhythmia, revealed that the neoplastic mass could affected the common bundle of His, in addition to the ordinary myocardium, being causative of circulatory failure $[18,19]$.

Although most cardiac rhabdomyomas have a relatively benign perinatal course, the long-term prognosis is determined by the neurological manifestations associated with tuberous sclerosis.

Rhabdomyoma may be detected prenatally by ultrasound in the third trimester of pregnancy. However, a large number is diagnosed in infancy. The diagnosis is suggested by clinical features of tuberous sclerosis and is made by echocardiography. Heart echocardiography, CT and MRI are the major non-invasive diagnostic procedures, while angiography is used in selected cases, due to its invasive nature. Even though ECG is not specific 
for the diagnosis, it is important to establish the presence of rhythm disorders, while a chest X-ray may show an enlarged heart silhouette or abnormal cardiac contours.

Therapeutic strategies should be individualised: the total resection is not the only therapeutic aim, in fact, the restoration of the regular heart function may be of primary relevance. Antiarrhythmic agents successfully control the clinical and electrophysiological conditions, while surgery is indicated in children with significant clinical symptoms. Benign cardiac tumours in childhood have an excellent prognosis when completely excised and have a good short-term prognosis even when excision is incomplete. Asymptomatic patients require anyway a close follow-up [20].

This report outlines the importance of an early diagnosis of cardiac tumours: when the prenatal and/or neonatal diagnosis of rhabdomyoma is made, an appropriate planning and an accurate follow-up may be performed to formulate individualised therapeutic strategies, improving in this way the clinical outcome and, eventually, anticipating the development of tuberous sclerosis.

\section{Consent}

Written informed consent was obtained from the patient's parents for publication of this case report and any accompanying images.

\section{Competing interests}

The authors declare that they have no competing interests.

\section{Authors' contributions}

VF contributed to this article and conceived the study. SDD and ET wrote the manuscript. RM and CP performed the macroscopic sections. MN and IR made the pathological explorations and performed the laboratoristic and microscopic analysis. All authors read and approved the final manuscript.

\section{Author details}

'Department of Forensic Pathology, University of Foggia, Ospedale Colonnello D'Avanzo, Via degli Aviatori 1, Foggia 71100, Italy. ${ }^{2}$ ASL BAT, Ospedale di Barletta, Barletta 76121, Italy. ${ }^{3}$ ASL, Ospedale di Matera, Matera 75100 , Italy.

Received: 29 October 2012 Accepted: 25 November 2012

Published: 3 December 2012

\section{References}

1. Castillo JG, Silvay G: Characterization and management of cardiac tumours. Semin Cardiothorac Vasc Anesth 2010, 14:6-20.

2. Freedom RM, Lee KJ, MacDonald C, Taylor G: Selected aspects of cardiac tumours in infancy and childhood. Pediatr Cardiol 2000, 21:299-316.

3. Stratemann S, Dzurik T, Fish F, Parra D: Left ventricular cardiac fibroma in a child presenting with ventricular tachycardia. Pediatr Cardiol 2008, 29:223-226.

4. Myers KA, Wong KK, Tipple M, Sanatani S: Benign cardiac tumours, malignant arrhythmias. Can J Cardiol 2010, 26:58-61.

5. Uzun O, Wilson DG, Vujanic GM, Parsons JM, De Giovanni JV: Cardiac tumours in children. Orphanet J Rare Dis 2007, 2:11.

6. Kohut J, Krzystolik-Ladzinska J, Szydlowski L, Smolenska-Petelenz J, GiecFuglewicz G, Pajak J: The diagnosis, clinical course and follow-up of children with cardiac tumours - a single - centre experience. Kardiol Pol 2010, 68:304-309.
7. Beghetti M, Gow RM, Haney I, Mawson J, Williams WG, Freedom RM: Paediatric primary benign cardiac tumours: a 15-year review. Am Heart J 1997, 134:1107-1114.

8. Becker AE: Primary heart tumours in the paediatric age group: a review of salient pathologic features relevant for clinicians. Pediatr Cardiol 2000, 21:317-323.

9. Burke A, Virmani R: Paediatric heart tumours. Cardiovasc Pathol 2008, 17:193-198.

10. Marx GR, Moran AM: Cardiac tumours. In Hearts disease in infants and adolescents. Edited by Moss AJ, Adams FH. Baltimore: Lippincott Williams \& Wilkins; 2008:1479-1495.

11. Sharma J, Inglis S, Cavalieri R, Udom-Rice I: Foetal demise secondary to massive rhabdomyoma in the early second trimester of pregnancy. Pediatr Cardiol 2011, 32:243-244.

12. Bader RS, Chitayat D, Kelly E, Ryan G, Smallhorn JF, Toi A, Hornberger LK: Foetal rhabdomyoma: prenatal diagnosis, clinical outcome, and incidence of associated tuberous sclerosis complex. J Pediatr 2003, 143:620-624.

13. Farooki ZQ, Ross RD, Paridon SM, Humes RA, Karpawich PP, Pinsky WW: Spontaneous regression of cardiac rhabdomyoma. Am J Cardiol 1991, 67:897-899.

14. Alkalay AL, Ferry DA, Lin B, Fink BW, Pomerance JJ: Spontaneous regression of cardiac rhabdomyoma in tuberous sclerosis. Clin Pediatr 1987, 26:532-535.

15. Kendziorra H, Kumpf M, Mackensen-Haen S, Bültmann B: Hypertrophic obstructive cardiomyopathy of an infant with Neuroblastoma: a case report. Diagn Pathol 2007, 2(Suppl 1):S20. 14 March 2007.

16. Bello S, Neri M, Riezzo I, Othman MS, Turillazzi E, Fineschi V: Cardiac beriberi: morphological findings in two fatal cases. Diagn Pathol 2011, 6:8.

17. Turillazzi E, Bello S, Neri M, Riezzo I, Fineschi V: Colloid cyst of the third ventricle, hypothalamus, and heart: a dangerous link for sudden death. Diagn Pathol 2012, 7:144.

18. Kitamura M, Wada N, Nagata S, lizuka N, Jin YF, Tomoeda M, Yuki M, Naka $\mathrm{N}$, Araki N, Yutani C, Tomita Y: Malignant peripheral nerve sheath tumor associated with neurofibromatosis type 1, with metastasis to the heart: a case report. Diagn Pathol 2010, 5:2.

19. Turillazzi E, Bello S, Neri M, Pomara C, Riezzo I, Fineschi V: Congestive heart failure as cause of death in an anorexia nervosa fatal case. Int J Cardiol 2012. doi:pii: S0167-5273(12)01443-X. 10.1016/j.ijcard.2012.10.068 [Epub ahead of print].

20. Qiu LS, Sun YJ, Ding WX, Xu ZW, Liu JF: Treatment strategies for paediatric patients with primary cardiac tumours. Zhonghua Wai Ke Za Zhi 2011, 49:227-231

doi:10.1186/1746-1596-7-169

Cite this article as: Neri et al.: Sudden death as presenting symptom caused by cardiac primary multicentric left ventricle rhabdomyoma, in an 11-month-old baby. An immunohistochemical study. Diagnostic Pathology 2012 7:169.

\section{Submit your next manuscript to BioMed Central and take full advantage of:}

- Convenient online submission

- Thorough peer review

- No space constraints or color figure charges

- Immediate publication on acceptance

- Inclusion in PubMed, CAS, Scopus and Google Scholar

- Research which is freely available for redistribution 